\title{
Relationship Between Euglycemic Diabetic Ketoacidosis and SGLT-2 Inhibitors
}

\author{
Mehmet Serdar Cengizhan ${ }^{1}$ and Mehmet Çelik ${ }^{2 *}$ \\ ${ }^{1}$ Department of Internal Medicine, Bilecik State Hospital, Turkey \\ ${ }^{2}$ Department of Endocrinology and metabolism, Bilecik State Hospital, Turkey \\ *Corresponding author: Mehmet Çelik, Department of Endocrinology and metabolism, Bilecik State Hospital, Turkey
}

Submission: 監April 02, 2018; Published: 㭗 April 27, 2018

\begin{abstract}
Diabetes mellitus is increasingly diagnosed today. In the treatment of diabetes mellitus, insulin and many oral anti-diabetic agents are used. With the increasing understanding of the pathophysiology of diabetes mellitus today, new pharmacological drugs are being developed and marketed for the use of patients in the treatment of diabetes. SGLT2 inhibitors from top-grade antidiabetic agents were used in the last decade for the use of patients diagnosed with diabetes and one of the newer generation drugs with different mechanisms of action. However, studies and case-reports suggesting that this drug group may increase the risk of euglycemic diabetic ketoacidosis. F.D.A (Food and Drug Administration) a warning was issued by May 2015. In this article we aimed to investigate the relationship between SGLT-2 inhibitors and diabetic ketoacidosis and to show that further data collection and study in this regard and appropriate follow-up are warranted.
\end{abstract}

Keywords: Euglycemic diabetic ketoacidosis; SGLT-2 inhibitors

\section{Introduction}

SGLT-2 inhibitors are a new class of drugs that lower blood sugar by inhibiting sodium-glucose cotransporter 2 in the treatment of type 2 diabetes. The HBA1C reduction effects of these agents are approximately $0.5-1.0 \%[1,2]$. These drugs inhibit SGLT-2 in the proximal nephron and these effects increase glucose excretion from urine up to $80 \mathrm{~g}$ /day by reducing endogenous glucose reabsorbtion $[2,3]$. Since this is insulin-dependent, SGLT2 inhibitors can be used in any type 2 diabetes mellitus, including patients in whom insulin secretion has been significantly reduced. Other additional benefits of this group of medicines are the weakening of the average 2 kilograms after 6-12 months of use in patients and a reduction of $2-4 \mathrm{mmHg}$ in systolic pressure and $1-2 \mathrm{mmHg}$ in diastolic pressure respectively $[1,2,4]$.

In addition, although the clinical significance of these medications is not yet known, serum uric acid levels and albuminuria have also decreased [5]. Diabetic ketoacidosis is a lifethreatening complication that can not be diagnosed and treated promptly in patients with diabetes. Diabetic ketoacidosis (DKA) tends to occur in poorly controlled type 1 diabetes patients with severe insulin insufficiency, but may also be seen in patients with Type 2 diabetes who are suffering from exogenous stress infection, trauma, or post-surgical disease.

Although DKA is typically presumed to be caused by elevated blood glucose and consequent dehydration, it is rarely seen when there is a slight increase in blood glucose in the DKA. This rare form of DKA is known as euglycemic or normoglycemic. DKA original definition is called with a blood glucose level $<300 \mathrm{mg} /$ $\mathrm{dl}$, but nowadays, cases of diabetic ketoacidosis in the presence of blood glucose level $<200 \mathrm{mg} / \mathrm{dl}$ have been described.Although the majority of euglycemic DKA cases are Type $1 \mathrm{DM}$, they rarely occur in Type 2 DM patients. Euglesmic DKA may also be seen in pregnant women, possibly due to altered ketogenic metabolism during pregnancy. SGLT2 inhibitors are Ipragliflozin, Dapagliflozin, Luseogliflozin, Tofogliflozin, Canagliflozin, and Empagliflozin.

Recently, eleven cases of diabetic ketoacidosis have been reported with slight or normal blood glucose level when using the SGLT-2 inhibitor Canagliflozin.Of these 9 patients, 7 were Type 1 diabetes mellitus patients who had been treated with diabetic ketoacidosis, which was 11 times. Six of the diabetic ketoacidosis episodes of these patients had blood glucose $<200 \mathrm{mg} / \mathrm{dl}$, four were blood glucose $250-300 \mathrm{mg} / \mathrm{dl}$, and the remaining one had no blood glucose level was undetermined. The other two case reports were Type 2 Diabetes Mellitus and blood glucose levels $<200 \mathrm{mg} / \mathrm{dl}$ when entering diabetic ketoacidosis and both patients had recently undergone a surgical operation [6].

Two patients with type 2 diabetes who were also taking an SGLT-2 inhibitor, Dapagliflozin, also reported euglycemic diacetic ketoacidosis. One of these two cases of euglycemic diabetic ketoacidosis was diagnosed as mucinous cystadenocarcinoma and distal pancreatomy was performed.This patient was being treated 
with insulin in the intensive care unit after surgery, but the insulin was used instead of the patient, and the SGLT-2 inhibitor was started. Diabetic ketoacidosis developed when blood glucose was $106 \mathrm{mg} / \mathrm{dl}$ in the patient 24 hours after this treatment change.Blood glucose value was $187 \mathrm{mg} / \mathrm{dl}$. In the second patient who developed diabetic ketoacidosis, no triggering factor was found, but there was pancreatic atrophy and pancreatitis story in this patient's story [7].

In Japan, 28 cases associated with the use of SGLT-2 inhibitor until 2015 have been reported. Seven of these cases had blood glucose level $<200 \mathrm{mg} / \mathrm{dl}$ [8], $200-299 \mathrm{mg} / \mathrm{dl}$ in two cases, and $>300$ $\mathrm{mg} / \mathrm{dl}$ in five cases, while blood glucose levels in the other 14 cases were not defined. In May 2015, F.D.A. has issued a warning that the use of an SGLT-2 inhibitor may increase the risk of ketoacidosis [9]. The study was terminated because of the development of diabetic ketoacidosis in two patients at an 8-week study with Empagliflozin containing 42 patients with type 1 diabetes $[10,11]$.

\section{Discussion}

It is difficult and early to comment that SGLT-2 inhibitors, which have not yet become very prevalent as of 2018, trigger normoglycemic or euglycemic diabetic ketoacidosis in the context of limited information and data available, and that these cases develop secondary to the SGLT-2 inhibitor. SGLT-2 inhibitors increase serum glucose levels by increasing glucose excretion during urination, which in time reduces insulin release from pancreatic beta cells. As a manifestation of decreased insulin levels in the body circulatory system, the antilipolytic activity of insulin is diminishing, leading to an increase in free fatty acid, which converts to ketone bodies in the liver. Moreover, by activating insulin acetyl-CoA carboxylase activity, Malonyl-CoA, a potent inhibitor of carnitine palmitoyl transferase-1 (CPT-I), is produced. CPT-I promotes the transport of fatty acids into mitochondria which increases the rate of beta oxidation. And it provides support for the production of ketone bodies due to CPT-I activation with reduced circulating insulin levels [12].

In addition, there is some evidence suggesting that the use of SGLT-2 inhibitor may stimulate glucagon release, glucagon inhibits acetyl CoA carboxylase, thereby increasing the likelihood of overproducing ketone bodies with increased CPT-I activity in the liver [13]. Evidence suggests that patients with Type 2 diabetes mellitus in East Asia, including Japan, that makes us think that it is weaker and its diseases are more attributable to the failure of the beta cell [14]. In this respect, it would be a more correct approach to consider that long-acting type 2 diabetes mellitus should be added to treatments after a more careful evaluation of prescribing of SGLT-2 inhibitors in diabetic patients, especially those of East Asian origin, whose beta cell function is thought to decrease over time. As a result, it is very difficult to establish a clear association between SGLT-2 inhibitors and diabetic ketoacidosis in the presence of diabetic ketoacidosis, but with further work to be done in this regard, it will be widespread in the coming years whether this group of drugs is associated with euglycemic diabetic ketoacidosis should be assessed in detail in the context of research.

\section{References}

1. Vasilakou D, Karagiannis T, Athanasiadou E, Mainou M, Liakos A, et al. (2013) Sodium-glucose cotransporter 2 inhibitors for type 2 diabetes: a systematic review and meta-analysis. Ann Intern Med 159(4): 262-274.

2. Stenlöf K, Cefalu WT, Kim KA, Alba M, Usiskin K, et al. (2013) Efficacy and safety of canagliflozin monotherapy in subjects with type 2 diabetes mellitus inadequately controlled with diet and exercise. Diabetes Obes Metab 15(4): 372-382.

3. Ferrannini E, Solini A (2012) SGLT2 inhibition in diabetes mellitus: rationale and clinical prospects. Nat Rev Endocrinol 8(8): 495-502.

4. Rosenstock J, Seman LJ, Jelaska A, Hantel S, Pinnetti S, et al. (2013) Efficacy and safety of empagliflozin, a sodium glucose cotransporter 2 (SGLT2) inhibitor, as add-on to metformin in type 2 diabetes with mild hyperglycaemia. Diabetes Obes Metab 15(12): 1154-1160.

5. Chino Y, Samukawa Y, Sakai S, Nakai Y, Yamaguchi J, et al. (2014) SGLT2 inhibitor lowers serum uric acid through alteration of uric acid transport activity in renal tubule by increased glycosuria. Biopharmaceutics \& Drug Disposition 35(7): 391-404.

6. Peters AL, Buschur EO, Buse JB, Cohan P, Diner JC, et al. (2015) Euglycemic diabetic ketoacidosis: a potential complication of treatment with sodium-glucose cotransporter 2 inhibition. Diabetes Care 38(9): 1687-1693.

7. Hine J, Paterson H, Abrol E, Russell Jones D, Herring R (2015) SGLT inhibition and euglycaemic diabetic ketoacidosis. Lancet Diabetes Endocrinol 3(7): 503-504.

8. Hayami T, Kato Y, Kamiya H, Kondo M, Naito E, et al. (2015) Case of ketoacidosis by a sodium-glucose cotransporter 2 inhibitor in a diabetic patient with a low-carbohydrate diet. J Diabetes Investig 6(5): 587-590.

9. US Food and Drug Administration (2015) FDA warns that SGLT2 inhibitors for diabetes may result in a serious condition of too much acid in the blood. FDA Drug Safety Communication.

10. Cherney DZ, Perkins BA, Soleymanlou N, Maione M, Lai V, et al. (2014) Renal hemodynamic effect of sodium-glucose cotransporter 2 inhibition in patients with type 1 diabetes mellitus. Circulation 129(5): 587-597.

11. Perkins BA, Cherney DZ, Partridge H, Soleymanlou N, Tschirhart H, et al. (2014) Sodium-glucose cotransporter 2 inhibition and glycemic control in type 1 diabetes: results of an 8-week open-label proof-of-concept trial. Diabetes Care 37(5): 1480-1483.

12. Ogawa W, Sakaguchi K (2016) Euglycemic diabetic ketoacidosis induced by SGLT2 inhibitors: possible mechanism and contributing factors. J Diabetes Investig 7(2): 135-138.

13. Kibbey RG (2015) SGLT-2 inhibition and glucagon: cause for alarm? Trends Endocrinol Metab 26(7): 337-338.

14. Møller JB, Pedersen M, Tanaka H, Ohsugi M, Overgaard RV, et al. (2014) Body composition is the main determinant for the difference in type 2 diabetes pathophysiology between Japanese and Caucasians. Diabetes Care 37(3): 796-804. 
GJEM Global Journal
of Endocrinological
Metabolism

For possible submissions Click Here
Submit Article
Global Journal of Endocrinological Metabolism

\section{Benefits of Publishing with us}

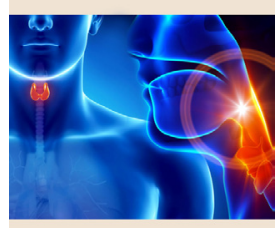

- High-level peer review and editorial services

- Freely accessible online immediately upon publication

- Authors retain the copyright to their work

- Licensing it under a Creative Commons license

- Visibility through different online platforms 